\title{
Imprese di perforazione e procedure amministrative per i pozzi
}

\author{
Marco Vannucchi \\ GEOSTUDI s.r.l. - Parma \\ m.vannucchi@geostudiparma.it
}

Nel particolare contesto di crisi economica quale quello attuale, che ha interessato e coinvolto tutti i settori produttivi, nessuno escluso, nell'ambito delle attività promosse dall'Associazione Nazionale Idrogeologia e Pozzi per Acqua e dall'Associazione Acque Sotterranee nel mese di giugno 2012 si è tenuta a Piacenza una riunione tra le ditte di perforazione emiliano-romagnole al fine di discutere ed affrontare le importanti problematiche connesse a tale settore, localmente ulteriormente aggravate dai recenti eventi sismici che hanno, di fatto, paralizzato l'attività produttiva di una parte della regione ove viene prodotto circa un $2 \%$ del pil nazionale.

Nell'incontro sono state affrontate, in particolare, le tematiche derivanti dalle tempistiche autorizzative da parte degli uffici periferici della Regione Emilia-Romagna inerenti la realizzazione di pozzi, specialmente ad uso extra-domestico, ove l'autorizzazione alla perforazione si configura titolo indispensabile per l'inizio dei lavori e per il successivo rilascio del titolo di concessione.

Generalmente, ma soprattutto in questo particolare momento, risulta quanto mai difficoltoso, da parte delle ditte di perforazione, come emerso in sede di confronto, sostenere tempistiche autorizzative dilatate, temporalmente parlando, talora prossime anche all'anno, che, di fatto, tendono a procrastinare nel tempo la realizzazione dei pozzi e quindi le possibilità di lavoro per le ditte stesse; tempi legati anche al rilascio dei pareri previsti per legge (art.12 RER n41/01 pareri di Autorità di Bacino, Provincia competente e se del caso, Enti Parco, AUSL, Consorzi di bonifica, Agenzie d'ambito e Servizio regionale competente per VIA) che, di fatto, talora impongono anche restrizioni o negazioni alla perforazione..

Dal confronto è emerso come tale situazione non sia tuttavia generalizzata su tutto il territorio regionale, pur operando nell'ambito della medesima regione ove l'iter normativo è regolato dal Regolamento regionale $\mathrm{n}^{\circ} 41$ del 22.11.2001 "Regolamento regionale per la disciplina del procedimento di concessione di acqua pubblica".

Sono state, inoltre, segnalate differenziazioni, tra le varie provincie, anche nella gestione relativa alle comunicazioni circa l'esecuzione di pozzi ad uso domestico (T.U. R.D. 1775/1933, art.93 - Reg.RER n41/01 art.3 let.p - opere non soggette ad autorizzazione e a concessione), sia per la parte amministrativa che per quella tecnica, sottolineando come per questo particolare tipo di perforazione non sia generalmente necessaria la presentazione di un vero e proprio progetto come viceversa avviene obbligatoriamente per le opere destinate ad uso extra-domestico (art.16 Reg.RER n41/01) .

Non è questa la sede opportuna per elencare e trattare la possibile casistica di merito, ma è sicuramente un importante momento di riflessione che deve coinvolgere non solo le ditte stesse ma tutto il sistema interessato, partendo dai professionisti, geologi, generalmente ed auspicabilmente progettisti delle opere, insieme al loro Ordine di appartenenza e gli Enti preposti al fine di trovare soluzioni tese, nel rispetto ovviamente delle normative vigenti, alla velocizzazione degli iter burocratici; ciò anche al fine di evitare il possibile dilagare delle perforazioni abusive con danno, oltre che erariale per il mancato incasso di canoni e relative spese accessorie, anche e soprattutto ambientale permanente per il mancato controllo di requisiti minimi di qualità.

In tale contesto riteniamo di fondamentale importanza le possibili azioni esercitate o esercitabili dal Geologo, generalmente e, come detto, auspicabilmente progettista delle opere di emungimento, nonché dall'Associazione, sia nei confronti degli Enti preposti al rilascio delle autorizzazioni che degli associati stessi.

Il Geologo, in quanto progettista, di fatto deve, già in fase progettuale, verificare la fattibilità dell'opera rispetto alla vincolistica sovraordinata regionale o locale (vedi Piano Regionale di Tutela delle Acque, Piani Territoriali di Coordinamento Provinciali, PAI, PSC ecc...) o evidenziarne possibili limitazioni nonché garantire, con una adeguata progettazione, standard qualitativi dell'opera, finalizzati sia al soddisfacimento delle richieste progettuali che alla tutela ambientale.

L'Associazione, in quanto garante di una intera categoria la quale, nel confronto, ha cercato di ottimizzare e migliorare le proprie conoscenze.

A tal fine è proprio per questo, ovvero per pervenire ad un continuo miglioramento che ANIPA, con propri rappresentanti, è presente al tavolo per la stesura delle norme UNI riguardanti non solo gli "Impianti geotermici a bassa temperatura con pompa di calore" ma anche, e soprattutto, in questo caso, i "Pozzi per acqua" (progettazione e costruzione).

L'intenzione è quella di innalzare il più possibile il livello qualitativo al fine di facilitare le attività istituzionali, sia autorizzative che di tutela ambientale, attraverso un recupero delle competenze e della professionalità.

Lobiettivo, a fronte quindi di elevati standard qualitativi, sia progettuali che realizzativi, sarà quello di coinvolgere e portare gli Enti preposti alla standardizzazione e, per quanto possibile, velocizzazione degli iter amministrativi, anche per mezzo di asseverazioni rilasciate da tecnici progettisti ed imprese, soprattutto per quelle zone non gravate da particolari problematiche vincolistiche, geologiche e/o ambientali . 